$10-2019$

\title{
Kisspeptin and attributes of infertile males and females: A cross- sectional study in a subset of Pakistani population
}

\author{
Rehana Rehman \\ Aga Khan University, rehana.rehman@aku.edu \\ Syeda Sadia Fatima \\ Aga Khan University, sadia.fatima@aku.edu \\ Faiza Alam \\ University of Karachi, Karachi, Pakistan \\ Mussarat Ashraf \\ Aga Khan University, mussarat.ashraf@aku.edu \\ shaheen zafar \\ Atia General Hospital, Malir, Karachi, Pakistan
}

Follow this and additional works at: https://ecommons.aku.edu/pakistan_fhs_mc_bbs

Part of the Physiology Commons

\section{Recommended Citation}

Rehman, R., Fatima, S. S., Alam, F., Ashraf, M., zafar, s. (2019). Kisspeptin and attributes of infertile males and females: A cross-sectional study in a subset of Pakistani population. Andrologia, 51(9), e13370.

Available at: https://ecommons.aku.edu/pakistan_fhs_mc_bbs/782 
Title:

2 Kisspeptin and attributes of infertile males and females: a cross sectional study in a subset of Pakistani Population

Running Title:

Infertility and Kisspeptin

Rehana Rehman, Syeda Sadia Fatima, Faiza Alam*, Mussarat Ashraf, Shaheen Zafar

Rehana Rehman: Associate Professor, Department of Biological and Biomedical Sciences, Aga Khan University, Stadium Road, Karachi, 74800, Pakistan.

Syeda Sadia Fatima: Assistant Professor, Department of Biological and Biomedical Sciences, Aga Khan University, Stadium Road, Karachi, 74800, Pakistan.

Faiza Alam: PhD scholar, Department of Physiology, University of Karachi, Main University Road, Karachi, Sindh 75270, Pakistan.

Mussarat Ashraf: Senior Technologist, Department of Biological and Biomedical Sciences, Aga Khan University, Stadium Road, Karachi, 74800, Pakistan

Shaheen Zafar: Medical Director, Sindh Institute of Reproductive Medicine. Atia General Hospital, Malir, Karachi, Pakistan.

\section{Corresponding Author:}

Dr. Faiza Alam

Department of Physiology,

University of Karachi, Karachi, Pakistan

Email: faiza.orakzai@gmail.com 

study in a subset of Pakistani Population.

Kisspeptin; a peptide hormone, plays a pivotal role in fertility and neuroendocrine regulation of hypothalamo-pituitary gonadal axis. Increased

\section{Introduction:}


51 Kisspeptin (KP) encoded by KISS1 belongs to a family of peptide hormones 52 which play a principal role in fertility and neuroendocrine regulation of 53 hypothalamo-pituitary gonadal axis (Vaziri, Rafeie et al. 2017). KP secreting 54 neurons are present in the different nuclei of hypothalamus; arcuate nucleus 55 (ARC) also known as infundibular nucleus, the anteroventral peri-ventricular 56 nucleus (AVPV), anterodorsal preoptic nucleus (APN), stria terminalis and 57 Amygdale (Funes, Hedrick et al. 2003). The pulsatile secretion of Gonadotropin Releasing Hormone (GnRH) in central regulation of the Hypothalamo-pituitary gonadal (HPG) axis is played by hypothalamic KISS1/KISS1R (receptor of KISS 1) system (Skorupskaite, George et al. 2014). Consequently, the cross-talk between Kisspeptin and the receptor $(K I S S 1 R)$ stands crucial in regulating the commencement of puberty and release of hormones from the involved reproductive axis (Luan, Zhou et al. 2007)

KISS1 translates for a 145 amino acid long protein identified as kisspeptin-145 which produces a peptide containing 54 residues after cleavage, called Kisspeptin 54 or metastin that can further be sliced into much smaller amino acid sequences, recognized as kisspeptin-14, kisspeptin-13 and kisspeptin-10, however they represent a common structural motif (ArgPhe-NH2) in their Cterminal (de Tassigny, Fagg et al. 2007). Along the length of gene KISS1, more than approximately 294 single nucleotide polymorphism (SNPs) are already identified; among which the untranslated region (UTR) contributes to have 42 mutations, exon for 30 and the rest by intronic regions. [“A database of human single nucleotide polymorphisms" 2014, http://www.ncbi.nlm.nih.gov/SNP/].

$K I S S 1 R$ gene mutations result in loss of function of the KISSIR, leading to down-regulation of GnRH pulsatile secretion as well as infertility. On the other 
76 hand, activating mutations cause prevention of desensitization of the 77 KISS/KISSIR pathway and ultimately lead to precocious puberty. Two KISS1 78 mutations, p.P74S and p.H90D, have been recognized as genetic causes of 79 Central Precocious Puberty (Silveira, Noel et al. 2010). Furthermore, 80 polymorphism of the KISS1 gene with amino acid substitution (P110T) The role of KP in feedback regulation of GnRH secretion and hence release of gonadal hormones required for normal reproductive functions has been elucidated (Irwig, Fraley et al. 2004). Evidence advocates the loss of gene functionality or presence of SNPs in KISS1 and KISSIR to be a risk factor for sexual immaturity and infertility axis in humans (Ko, Lee et al. 2010).

A number of studies have verified role of Kisspeptin on reproductive axis, unexplained infertility and as a therapeutic agent to trigger oocyte maturation and ovulation (Abbara, Jayasena et al. 2014), (Mumtaz, Khalid et al. 2017). Literature has proved that mutations which inhibit the action of KISS1 and KISSIR in idiopathic hypo-gonadotropic-hypogonadism (IHH) subjects resulted in delayed puberty and subfertility (Semple, Achermann et al. 2005) .

Although mutations inhibiting KISS1 gene and its receptor activity can instigate infertility yet, the information about KISS1 gene mutations and its polymorphisms are scarce. A research done on Q36R (rs35431622) KISS1 gene in infertile female subjects in northern Iran documented that it has no association with female infertility and suggested that variation of results might be possible due to genetic variations on account of different geographic situations (Vaziri, 
We thus aimed to explore role of KP on HPG axis by comparing KP levels in fertile and infertile male and female subjects and identify the sequence variations, including mutations and single-nucleotide polymorphisms (SNPs) of KISS1 gene in exon 2 and 3 of infertile males and female cohorts.

This cross-sectional study was conducted in the Department of Biological \&

Biomedical Sciences, Aga Khan University, Karachi, from April 2016 till March

2018 after acquiring ethical approval (3331-BBS-ERC-14). The estimated sample

size by observing $94 \%$ power, with prevalence of $21.9 \%$ infertility and $22 \%+/-6$

confidence limit, was 79. To avoid drop out of subjects we recruited 88 infertile

inclusion criteria and consented to be part of our study.

\section{Phenotypic Characterization:}

116 All males between the ages of 25 to 55 years during the study phase who

117 concurred to take part in the study were selected. A comprehensive history

118 related to the diagnosis of infertility was obtained for excluding secondarily

119 infertile males. A semen analysis report was obtained on request (6 to 9 months 120 old). In case of failure to do so, male subjects were demanded a fresh semen 121 samples by masturbation following a 3 - 5 days of asceticism. Samples were then 122 processed and stored in sterilized containers, later were analyzed as per World $123 \quad$ Health Organization guidelines. 
125 The "fertility status" of the recruited subjects was established according to the 126 semen parameters observing the World Health Organization criteria "2010" 127 which states "had total sperm number (TC) >39 million per ejaculate, total sperm 128 motility (Progressive and Non-progressive) measured within 60 minutes of 129 collection of more than $40 \%$, and normal morphology of $\geq 4 \%$." (Cooper, $130 \quad$ Noonan et al.)

Infertile group:

Men with history of primary infertility; sperm number (TC) less than 39

133 million/ejaculate, decreased sperm motility of all sperms (less than 40\%) and 134 having normal sperm morphology of less than $4 \%$ (Cooper, Noonan et al. 2010) 135 were included in the study.

\section{Exclusion Criteria for Male Subjects:}

137 Subjects having diabetes, hypertension, arthritis, malignancy, epilepsy, 138 tuberculosis, endocrinal disorders, liver/renal disease, cryptorchidism, testicular 139 trauma, orchitis, testicular hypotrophy along with those who had general health 140 issues were excluded. Additionally, those who suffered from secondary 141 infertility were discounted. Moreover, subjects who were receiving any 142 hormonal or steroids therapy were also excluded.

$143 \quad$ Inclusion Criteria for Female Subjects:

$144 \quad$ Fertile females:

145 All healthy females between the ages of $18-35$ years, with a child less than 2 years of age from all ethnic groups were recruited as controls. 
148 All females who fulfill the criteria of primary infertility (never conceived in last

149 more than one years) between the ages of 18 - 35 years, from all ethnic 150 backgrounds, were enrolled in the study as cases.

152 Exclusion criteria for Female subjects:

153 The females who were diagnosed with secondary infertility, being treated with 154 oral contraceptive pills, having thyroid disorders, preexisting diabetes and 155 hypertension were excluded from the study.

156 Clinical Data Collection: The clinical data including age, height, weight, blood 157 pressure, menstrual/obstetric and gynecological history with general physical 158 examination was recorded in all study subjects. The height in centimeters 159 (converted to meters) and weight in kilograms of all the recruited subjects were 160 noted to calculate the body mass index (BMI), and categorized consulting 161 cutoffs for Asians, where $18-22.9 \mathrm{~kg} / \mathrm{m}^{2}$ was normal weight, overweight 23 $16224.9 \mathrm{~kg} / \mathrm{m}^{2}$ was overweight and BMI $\geq 25 \mathrm{~kg} / \mathrm{m}^{2}$ was considered obese (WHO 163 2004). Gender, age and height of every participant was entered manually into 164 the BIA machine by a digital keyboard, and it immediately revealed the 165 percentage fat mass (\% FM) of the individual (Lazzer, Boirie et al. 2003)

\section{Biochemical Measurement}

167 Serum samples were used to detect the hormones levels using commercially 168 available Enzyme Linked Immuno Sorbent Assay (ELISA) kits, following the 169 manufacturer's protocol. Follicle stimulating hormone (FSH) by Human FSH 170 Enzyme Immunoassay (Kit Cat. No DKO010; DiaMetra), LH by Human LH 171 Enzyme Immunoassay (Kit Cat. No DKO010; Dia Metra). Immunoassay for 
$\mathrm{FSH}$, the inter assay coefficient of variation, $<8 \%$ and intra assay coefficient of

173 variation, $<9.7 \%$ and similarly LH immunoassay, the inter assay coefficient of

174 variation was $<7.91 \%$; intra assay coefficient of variation was $<9.21 \%$. Serum

175 KP was measured by ELISA kit (Cat. No: 95611, Glory BioScience, USA). The

176 analytical sensitivity was $10.16 \mathrm{ng} / \mathrm{L}$ and intra and inter assay coefficients of

177 variation was less than $10 \%$ and $12 \%$, respectively. For assessing total

178 testosterone levels commercially available Human Total Testosterone (TT)

179 immune-enzymatic kit for serum analysis was utilized (Cat. No DKO002 by

180 Diametra).

181 Genotype Characterization:

182 Blood Sampling and genotyping:

183 Ten ml of venous blood was collected form all study subjects. DNA was isolated 184 from the leukocytes in the peripheral blood of the study subjects using a DNA 185 isolation kit (Genomic DNA Purification Kit Cat. No A1125 by Promega, USA). 186 The isolated DNA was quantified by measuring the ultraviolet (UV) absorbance 187 and determining the absorbance ratio (A280/A260) for $2 \mu \mathrm{L}$ samples, employing a 188 Nanodrop-ND1000 (Thermo Fisher Scientific, Waltham, MA). Extracted DNA was 189 considered pure at an absorbance ratio of $\sim 1.8$. Furthermore, gel electrophoresis was run to visualize the PCR products on $5 \mu \mathrm{L}$ of sample in $2 \%$ agarose gel against a $100 \mathrm{bp}$ ladder on approximately $15 \%$ of all samples for confirmation. Gel was observed in Gel Doc Imaging system (Biorad, United Kingdom) (Figure 1a \& b).

193 For exon 2: Polymerase chain reaction (PCR) was executed using the 2X PCR 194 Hotstart Master Mix (Cat\# G906, ABM (Applied Biological Materials Inc, Canada) 195 according to the instructions mentioned on the provided manual. The cycle 196 conditions during PCR were: 1 cycle for $5 \mathrm{~min}$ at $95^{\circ} \mathrm{C}$ for initial denaturation 

seconds, followed by a final extension of $1 \mathrm{~min}$ at $72{ }^{\circ} \mathrm{C}$.

199 For exon 3: PCR was executed employing the Go Taq (R) Hotstart Green Master 200 mix (Cat \#M5122, Promega Corporation, USA) according to the instructions mentioned on the provided manual. The cycle conditions during PCR were: 1 cycle for $5 \mathrm{~min}$ at $95{ }^{\circ} \mathrm{C}$ for initial denaturation followed by 35 cycles at $95{ }^{\circ} \mathrm{C}$ for $30 \mathrm{~s}$, $58^{\circ} \mathrm{C}$ for $45 \mathrm{~s}, 72{ }^{\circ} \mathrm{C}$ for $45 \mathrm{~s}$ followed by a final extension of $10 \mathrm{~min}$ at $72{ }^{\circ} \mathrm{C}$.

204 Purification of the PCR products was done using PCR Clean Up for DNA 205 Sequencing (Cat. No BT5100, Bio Basic Inc, Canada) following the manufacturer 206 protocol. Genotypic analysis was performed to detect mutations responsible for 207 infertility by PCR amplification of the fertile and infertile male $(n=80)$ and female 208 subjects $(\mathrm{n}=88)$ within the region of exon 2 (214bp) and exon 3 (606bp) of KISS1 209 gene. The obtained sequences were directly compared to previously published 210 KISS1 gene sequence using the MEGABLAST search tool in the National Center 211 for Biotechnology Information (NCBI) database. Sequence files were imported into 212 Chromas Lite, and then assembled using Molecular Evolutionary Genetic Analysis 213 version 6.0.

214 All coding exons (exons 2 and 3) of KISS 1 gene were PCR amplified with specific 215 primers as follows:

\begin{tabular}{|l|c|c|}
\hline & Forward primer $\left(5^{\prime}\right.$ to $\left.3^{\prime}\right)$ & Reverse primer $\left(3^{\prime}\right.$ to $\left.5^{\prime}\right)$ \\
\hline exon 2 & CAGATCCTGTGCCTGACCT & CCCACTCCTTTCCCCAGAG \\
& A & \\
\hline
\end{tabular}




\begin{tabular}{|l|l|l|}
\hline exon 3 & ATGGGATGACAGGAGGTGT & ACCATCCATTGAGGATGGA \\
& TG & AG \\
\hline
\end{tabular}

217 Statistical Analysis: Statistical analyses were performed using IBM Statistical

218 Package for the Social Sciences (IBM SPSS version 21; IBM Corp Inc, Armonk,

219 NY). Continuous variables (such as age, LH, FSH etc.) were represented as Mean

$220 \pm$ standard deviation and the evaluation of the categorical variables (BMI) was

221 expressed in terms of frequencies and percentages. To compare continuous and

222 categorical variables, Independent sample t-test and Pearson's chi square test was

223 applied. Correlations were adjusted for age and BMI for hormonal associations

224 (logistic regression). SNP data was calculated by chi-squared statistics, Odds ratio

225 with 95\% confidence interval was calculated for genotype and allele frequency

226 analysis.

$227 \quad$ Results:

228 The results of the study showed that infertile males were obese and were at a

229 lower age bracket as compared to fertile group $(\mathrm{p}<0.001)$. Furthermore, the

230 infertile females also demonstrated a higher BMI as compared to the fertile

231 females $(\mathrm{p}<0.001)$. The sperm count and motility was decreased along with

232 increased abnormal morphology in the infertile males as compared to the fertile

233 males $(\mathrm{p}<0.01) \quad($ Table 1$)$. A similar trend in terms of hormonal profile was

234 observed in the infertile female subjects; however, no difference for age was

235 recorded (Table 1). Comparison of hormones in Table 2 show significantly high

236 levels of KP, FSH, LH and Testosterone in fertile male, however KP, LH and

$237 \quad$ Estradiol levels were significantly higher in fertile female subjects with low FSH

238 levels. When tested for the correlations of Kisspeptin levels on the male

239 hormones of hypothalamo-hypophyseal-gonotrophic axis; FSH showed 
240 moderate positive relationship with KP levels $(\mathrm{r}=0.67 ; \mathrm{p}=<0.001)$, while 241 Testosterone $(\mathrm{r}=0.38 ; \mathrm{p}=<0.01)$ showed weak correlation with fertility in all 242 subjects. Similarly, KP showed weak positive correlation with Estradiol $243(\mathrm{r}=0.466 ; \mathrm{p}=0.001)$ while no relationship was observed with LH and FSH. All 244 correlations were lost when adjusted for age and BMI $(p>0.05)$.

245 Table 3 shows the genotype distribution of the polymorphic EXON 2 and 3 246 sequences. This study was able to identify one polymorphism in exon2 [E1225K 247 (G/A 3673)] and 3 unique polymorphisms in exon3 [P1945A (C/G 5833); 248 Insertion of $\mathrm{T}$ at $6075 ; \mathrm{G} 2026 \mathrm{G}(\mathrm{C} / \mathrm{G}$ 6078)] in the study population. 249 Interestingly, these polymorphisms were observed in higher frequency in 250 infertile group (both genders) versus the fertile; yet the presence or absence of 251 polymorphic site in both exons of interest failed to reveal any significant 252 difference in this study population $(\mathrm{p}>0.05)$.

253 This study documented one novel result in the study cohort. In a sample of $n=80$ 254 males and $n=88$ females; 03 subjects in each sex group tested positive for all 03 255 of the polymorphisms in exon-3 region. When their data was linked with the 256 polymorphic status; it was observed that 04 out of 06 individuals (male female 257 combined) were smokers with low KP and its related hormonal levels at various 258 points during the study. Furthermore, their sperm parameters fell in the abnormal 259 category (males) or attempt at Intracytoplasmic Sperm injection (ICSI) was 260 unsuccessful (females) (Table 4 and 5). This result shows a probable effect of 261 environmental changes on genetic alterations in the KP gene and its secondary 262 effect on the reproductive axis. 
The relationship of KP with the interruption of hypothalamic-pituitary-gonadal axis can be demonstrated by high concordance of the phenotypes between comparable genetic variants present in GnRH receptor, FSH and its receptor, $\mathrm{LH}$ and its receptor in mice and humans (Rehman, Jamil et al. 2015). In male gonadotropin and sex steroid hormone levels, which is comparable to studies in male infertile subjects(Haris Ramzan, Ramzan et al. 2015). This can probably be an explanation of KP role in preservation of spermatogenesis and hence fertility. Low FSH levels was observed in normozoospermic and azospermic infertile male subjects by Ramzan et al which was not significantly different in fertile and infertile males (Haris Ramzan, Ramzan et al. 2015). The significant low FSH levels in infertile male subjects of our study may be explained by lack of stratification of subjects into infertile categories on the basis of sperm parameters. In the female infertile subjects a high FSH explains the negative feedback interplay on HPG axis due to decrease in Estradiol secretion. Literature also supports a raised FSH in infertile females (Prasad, Parmar et al. 2015).

Kisspeptin is now safely and successfully used in both healthy and infertile human subjects after trials in United Kingdom, and it is possible that in the future the Kisspeptin signaling may be used as a target in the treatment of reproductive disorders (Hameed, Jayasena et al. 2011).

The role of Kisspeptin injections to stimulate the secretion of LH and FSH in numerous mammalian species including rats, mice, sheep, cows and monkeys is supportive of our statement (Navarro, Castellano et al. 2005). Furthermore, a study done by Dhillo et al 2015, documents that KP infusion significantly increased plasma LH, FSH, and testosterone levels (Clarke, Dhillo et al. 2015) 
is supportive of our study in which infertile study subjects had low KP, LH,

290 FSH and testosterone in male subjects. The correlation of KP with estradiol in

291 both genders explains that KP requires estradiol secretion to stimulate $\mathrm{GnRH}$

292 secretion as suggested by the study in which ovariectomy abolished the KP-

293 induced GnRH release in pubertal monkeys, and estradiol replacement resulted

294 in partial recovery of KP- induced GnRH release (Guerriero, Keen et al. 2012).

295 Kisspeptin represents and should be investigated in the treatment of fertility

296 disorders characterized by low gonadotropins or anovulation. (Clarke, Dhillo et

$297 \quad$ al. 2015)

298 Higher KP in non-obese males and non-obese young females were observed in 299 our study, which might be due to the fact that KP effects negatively on body 300 weight and calorie consumption (Walewski, Ge et al. 2014, Stengel 2011 \#214, 301 (Lin, $2015 \# 216$ ). BMI and body fat \% age were noted to be high in infertile 302 groups while KP was low which is perhaps due to certain changes in the sex 303 hormones which regulates obesity by increasing the serum triglycerides levels 304 (Shamai, Lurix et al. 2011, Kołodziejski, Pruszyńska-Oszmałek et al. 2018). It 305 is a well-established fact that there are various mechanisms how obesity causes 306 infertility (Talmor and Dunphy 2015).There is an ample evidence that KP 307 signaling tends to decrease the metabolic rate and initiates glucose intolerance 308 and increased body fat (Holmes 2014).

309 In this study, no clear-cut difference was observed in the genetic mutations 310 amongst fertile and infertile males and female subjects. Three unique KISS1 311 mutations were identified all together in unrelated subject in each gender 312 category. The absence of this variant in the fertile female group suggests that 313 this is a rare mutation which has a major qualitative effect on the KISS gene. 
314 However only 1 fertile male tested positive for this mutation; when we checked

315 the paternal age and status of fertility in the last 5 years, it was identified that

316 this gentleman was 33-year-old and had a baby 4 years ago. Perhaps this

317 substitution mutation is related to the age group and environmental or stress

318 related changes. There are examples in literature where same SNPs resulted in

319 diverse expressions. As an example in Chinese population a SNP of amino acid

320 substitution (P110T) in KISS1 in females with CPP was found to be statistically

321 interconnected to infertility whereas in Korean girls the same mutation had a

322 protective effect on infertility (Luan, Zhou et al. 2007) (Ko, Lee et al. 2010).

323 Yet, no data is available from the current literature to support this claim.

324 Therefore, more work is required to assess the functionally or causality of this

325 mutation with infertility.

326 In terms of mutations in exon 3, we found no link of the SNP's with infertility.

327 These mutations i.e. insertion of Thiamine at position 6075 and substitution of

328 Proline to Alanine at position 1945 may be explained as due to faulty gene

329 regulation process related with age, since all these individuals were in the age range

330 of 34 to 48 year. Interestingly, in the fertile group with these mutations; one female

331 had delivered a baby within last 7 months. This finding suggests that silent

332 mutations at these positions, does not affect the functional role of Kisspeptin

333 protein. The study is limited in terms of being a uni-centric study with a small

334 sample size in which the impact of polymorphism in infertile females on the basis

335 of cause of infertility has not been taken into consideration. Furthermore, the

336 association of gene variation of KISS1 could have been further validated in terms

337 of impact on different altered sperm parameters. 

effect relationship of Kiss1 gene variation with hormones of reproduction and

341 Conclusion: Role of KP in regulation of normal reproductive functions can be 342 explained on the basis of its effect on secretion of gonadotropins and sex steroids. 343 Polymorphism in exon2 [E1225K (G/A 3673)] and 3 unique polymorphisms in 344 exon3 [P1945A (C/G 5833); Insertion of T at 6075; G2026G (C/G 6078)] can be 345 further explored as plausible cause of decreased KP production in infertile male and 346 female subjects. Further detailed studies are warranted for understanding of the 347 mechanistic role of genetic variations of KP in infertility.

\section{Acknowledgement:}

349 We are extremely thankful to Pakistan Health Research Council for the support 350 of funds; 4-22-27/15/RDC/AKU along with ACIMC for subject recruitment. 351 Further, all the authors would like to acknowledge Dr. Zehra Jamil and Uroosa 352 Tariq for their valuable help during the research. 
355

356

357

358

359

360

361

362

363

364

365

366

367

368

369

370

371

372

373

374

375

376

377

378

379

380

381

382

383

384

385

386

387

388

389

390

391

392

393

394

395

396

397

398

399

400

401

402

Abbara, A., C. Jayasena, A. Comninos, M. Nijher, G. Christopoulos, C. Izzi-Engbeaya, M. Sridharan, S. Narayanaswamy, D. Ashby and M. Ghatei (2014). "Kisspeptin: a novel physiological trigger for oocyte maturation in in-vitro fertilisation treatment." The Lancet 383: S17.

Clarke, H., W. S. Dhillo and C. N. Jayasena (2015). "Comprehensive review on kisspeptin and its role in reproductive disorders." Endocrinology and Metabolism 30(2): 124-141.

Cooper, T. G., E. Noonan, S. Von Eckardstein, J. Auger, H. Baker, H. M. Behre, T. B. Haugen, T. Kruger, C. Wang and M. T. Mbizvo (2010). "World Health Organization reference values for human semen characteristics." Human reproduction update 16(3): 231-245.

de Tassigny, X. d. A., L. A. Fagg, J. P. Dixon, K. Day, H. G. Leitch, A. G. Hendrick, D. Zahn, I. Franceschini, A. Caraty and M. B. Carlton (2007). "Hypogonadotropic hypogonadism in mice lacking a functional Kiss1 gene." Proceedings of the National Academy of Sciences 104(25): 10714-10719.

Fatima, S. S., R. Rehman and B. Chaudhry (2014). "Body mass index or body fat! which is a better obesity scale for Pakistani population?" JPMA: Journal of the Pakistan Medical Association 64(11): 1225.

Funes, S., J. A. Hedrick, G. Vassileva, L. Markowitz, S. Abbondanzo, A. Golovko, S. Yang, F. J. Monsma and E. L. Gustafson (2003). "The KiSS-1 receptor GPR54 is essential for the development of the murine reproductive system." Biochemical and biophysical research communications 312(4): 1357-1363.

Guerriero, K. A., K. L. Keen, R. P. Millar and E. Terasawa (2012). "Developmental changes in $\mathrm{GnRH}$ release in response to kisspeptin agonist and antagonist in female rhesus monkeys (Macaca mulatta): implication for the mechanism of puberty." Endocrinology 153(2): 825-836.

Hameed, S., C. N. Jayasena and W. S. Dhillo (2011). "Kisspeptin and fertility." Journal of Endocrinology 208(2): 97-105.

Haris Ramzan, M., M. Ramzan, F. Ramzan, F. Wahab, M. Jelani, M. Aslam Khan and M. Shah (2015). "Insight into the Serum Kisspeptin Levels in Infertile Males." Archives of Iranian Medicine (AIM) 18(1).

Holmes, D. (2014). "Metabolism: kisspeptin signalling linked to obesity." Nat Rev Endocrinol 10(9): 511.

Irwig, M. S., G. S. Fraley, J. T. Smith, B. V. Acohido, S. M. Popa, M. J. Cunningham, M. L. Gottsch, D. K. Clifton and R. A. J. N. Steiner (2004). "Kisspeptin activation of gonadotropin releasing hormone neurons and regulation of KiSS-1 mRNA in the male rat." 80(4): 264272.

Ko, J. M., H. S. Lee and J. S. Hwang (2010). "KISS1 gene analysis in Korean girls with central precocious puberty: a polymorphism, p. P110T, suggested to exert a protective effect." Endocrine journal 57(8): 701-709.

Kołodziejski, P. A., E. Pruszyńska-Oszmałek, E. Korek, M. Sassek, D. Szczepankiewicz, P. Kaczmarek, L. Nogowski, P. Maćkowiak, K. W. Nowak and H. Krauss (2018). "Serum levels of spexin and kisspeptin negatively correlate with obesity and insulin resistance in women." Physiological research 67(1): 45-56.

Lazzer, S., Y. Boirie, M. Meyer and M. Vermorel (2003). "Evaluation of two foot-to-foot bioelectrical impedance analysers to assess body composition in overweight and obese adolescents." British Journal of Nutrition 90(05): 987-992.

Luan, X., Y. Zhou, W. Wang, H. Yu, P. Li, X. Gan, D. Wei and J. Xiao (2007). "Association study of the polymorphisms in the KISS1 gene with central precocious puberty in Chinese girls." European Journal of Endocrinology 157(1): 113-118. 
404 potential factor for unexplained infertility and impaired embryo implantation." 405 International journal of fertility \& sterility 11(2): 99.

406 Navarro, V., J. Castellano, R. Fernandez-Fernandez, S. Tovar, J. Roa, A. Mayen, R. 407 Nogueiras, M. Vazquez, M. Barreiro and P. Magni (2005). "Characterization of the potent 408 luteinizing hormone-releasing activity of KiSS-1 peptide, the natural ligand of GPR54." 409 Endocrinology 146(1): 156-163.

410 Prasad, B., D. Parmar, N. J. I. J. O. M. R. Sharma and H. Sciences (2015). "A study on serum 411 FSH, LH and prolactin levels among infertile women." 4(4): 876-878.

412 Rehman, R., Z. Jamil, S. S. Fatima and F. Alam (2015). "Silent Mutation in KISS1and KISS1R 413 and Unexplained Infertility."

414 Semple, R., J. Achermann, J. Ellery, I. Farooqi, F. Karet, R. Stanhope, S. O'rahilly and S. 415 Aparicio (2005). "Two novel missense mutations in g protein-coupled receptor 54 in a 416 patient with hypogonadotropic hypogonadism." The Journal of Clinical Endocrinology \& 417 Metabolism 90(3): 1849-1855.

418 Shamai, L., E. Lurix, M. Shen, G. M. Novaro, S. Szomstein, R. Rosenthal, A. V. Hernandez 419 and C. R. Asher (2011). "Association of body mass index and lipid profiles: evaluation of a 420 broad spectrum of body mass index patients including the morbidly obese." Obesity 421 surgery 21(1): 42-47.

422 Silveira, L. G., S. D. Noel, A. P. Silveira-Neto, A. P. Abreu, V. N. Brito, M. G. Santos, S. D. C. 423 Bianco, W. Kuohung, S. Xu and M. Gryngarten (2010). "Mutations of the KISS1 gene in 424 disorders of puberty." The Journal of Clinical Endocrinology \& Metabolism 95(5): 22764252280.

426 Skorupskaite, K., J. T. George and R. A. Anderson (2014). "The kisspeptin-GnRH pathway 427 in human reproductive health and disease." Human reproduction update 20(4): 485-500.

428 Talmor, A. and B. Dunphy (2015). "Female obesity and infertility." Best Practice \& 429 Research Clinical Obstetrics \& Gynaecology 29(4): 498-506.

430 Vaziri, H., A. Rafeie and Z. Siapoosh (2017). "Q36R (rs 35431622) Polymorphism in KISS1 431 Gene and Idiopathic Female Infertility in a Northern Iranian Population." Gene, Cell and 432 Tissue 4(3).

433 Walewski, J. L., F. Ge, H. Lobdell Iv, N. Levin, G. J. Schwartz, J. R. Vasselli, A. Pomp, G. Dakin 434 and P. D. Berk (2014). "Spexin is a novel human peptide that reduces adipocyte uptake of 435 long chain fatty acids and causes weight loss in rodents with diet-induced obesity." 436 Obesity 22(7): 1643-1652.

437 WHO, E. C. (2004). "Appropriate body-mass index for Asian populations and its 438 implications for policy and intervention strategies." Lancet 363: 157-163. 